\title{
Increase of the Dynamic Range of Catchup Experiments by High-Pass Filtering
}

\author{
D.J. Erskine
}

This paper was prepared for submittal to the 1995 APS Topical Conference

Seattle, WA

August 13-18, 1995

August 1995

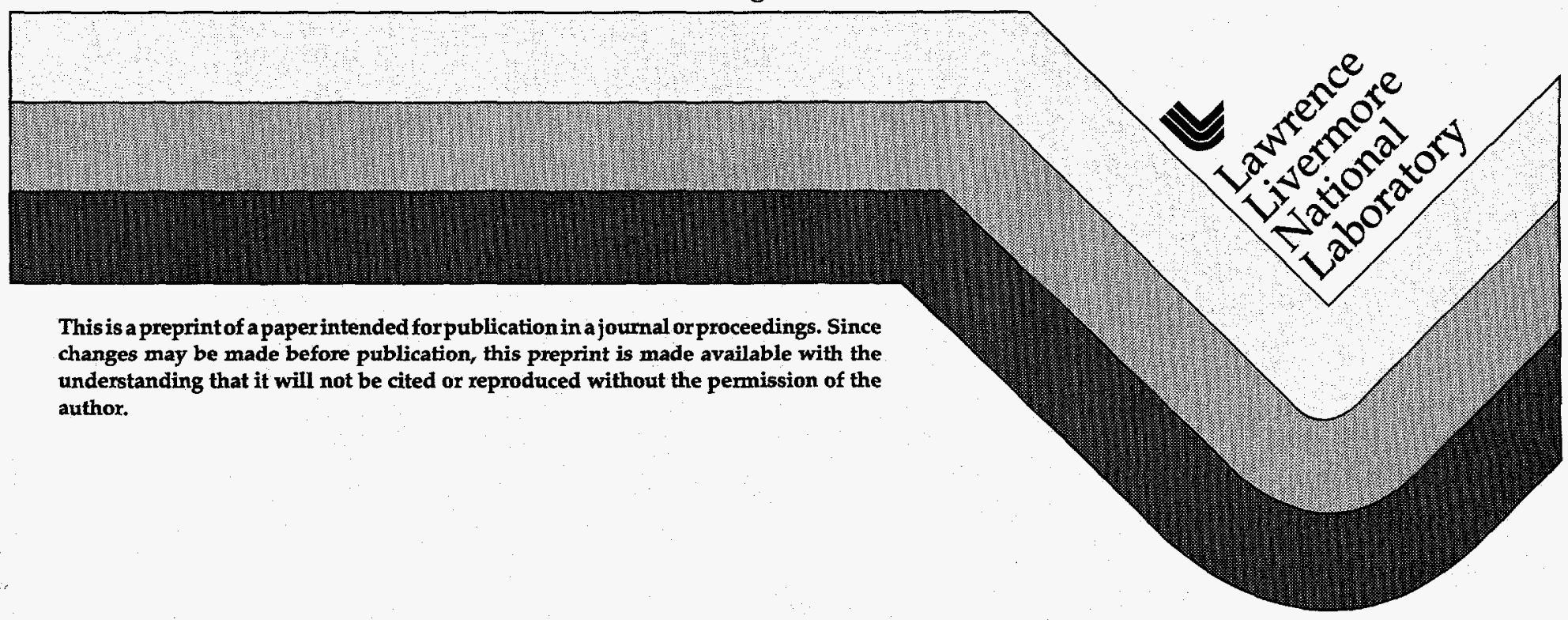


DISCLAIMER

This document was prepared as an account of work sponsored by an agency of the United States Government. Neither the United States Government nor the University of California nor any of their employees, makes any warranty, express or implied, or assumes any legal liability or responsibility for the accuracy, completeness, or usefulness of any information, apparatus, product, or process disclosed, or represents that its use would not infringe privately owned rights. Reference herein to any specific commercial product, process, or service by trade name, trademark, manufacturer, or otherwise, does not necessarily constitute or imply its endorsement, recommendation, or favoring by the United States Government or the University of California. The views and opinions of authors expressed herein do not necessarily state or reflect those of the United States Government or the University of California, and shall not be used for advertising or product endorsement purposes. 


\section{DISCLAIMER}

Portions of this document may be illegible in electronic image products. Images are produced from the best available original document. 


\title{
INCREASE OF THE DYNAMIC RANGE OF CATCHUP EXPERIMENTS BY HIGH-PASS FILTERING
}

\author{
David J. Erskine \\ Lawrence Livermore National Laboratory, Livermore, CA 94551
}

The release-catchup shock experiment is an important tool for measuring the speed of sound in compressed matter. The catchup of the release wave to the leading shock is sensitively detected optically, through an indicating fluid which produces light approximately to the 4th power of the shock pressure. However, this sensitivity demands a dynamic range which exceeds the capabilities of our digitizer. The catchup signature lies at the top of a flat pulse, thus any signal clipping is a catastrophic loss of data. We have invented a simple and accurate method for recording the catchup signature that is insensitive to signal clipping. A high pass circuit prior to the digitizer is used with post experiment integration. The insensitivity to clipping allows recording the catchup signature at higher gain, and thus with an improved signal to noise ratio.

\section{INTRODUCTION}

An important kind of shock experiment is the "release-catchup". experiment promoted by McQueen et al. ${ }^{1-3}$, used to measure the speed of sound in compressed materials. In this experiment, a thin impactor generates a shock and release wave both traveling forward through the sample. The depth at which the release catches up to the leading shock is a measure of the sound speed in the compressed sample. The moment of catchup is detected most sensitively optically, from behind the target. Behind the sample is a fluid which produces light under shock. When the shock enters the fluid the light intensity jumps immediately to a nearly constant level (Fig. 1).

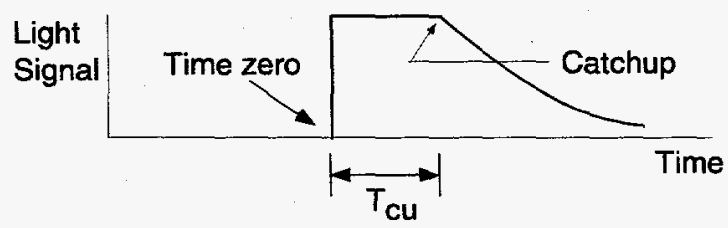

Figure 1. Shock entering liquid generates light related to pressure by $I \sim P^{4}$. When the rarefaction wave catches up to leading shock the intensity changes slope. Time from start of light to catchup $\left(T_{c u}\right)$ is the measurement of interest.

DISTAIBUTION OF THIS DOCUMENT IS UNLMITED
When the release wave catches up to the leading shock in the fluid, the light intensity drops sharply, producing a change in slope denoted the catchup point. Measuring the time difference $T_{c u}$ between the start of the light pulse and the catchup point is the goal of the experiment.

a)

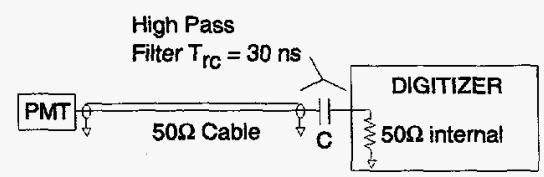

b)

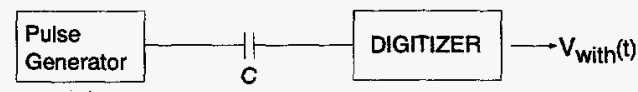

c)

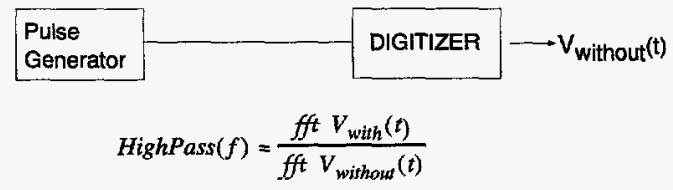

Figure 2. a) Electrical configuration. Photomultiplier (PMT) generates signal from shock generated light. Capacitor and internal $50 \Omega$ impedances of recorder and cable form a high pass filter with time constant $\mathrm{T}_{\mathrm{rC}}=\mathbf{3 0} \mathrm{ns}$. Transfer function of filter HighPass(f) measured by applying test pulse with b) and without c) filter and dividing their Fourier transforms $(f f t)$.

McQueen et al report ${ }^{2}$ that the light intensity I varies with the impactor velocity $\mathrm{U}_{\mathrm{f}}$ approximately

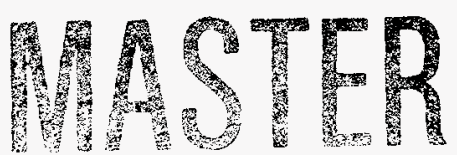




$$
\mathrm{I} \sim \mathrm{U}_{\mathrm{f}}^{8} .
$$

This is consistent with $\mathrm{I} \sim \mathrm{T}^{4}$ by the StefanBoltzmann law, and typically observed shock behaviors $T \sim P$, and $P \sim U_{f}{ }^{2}$, where $T$ is temperature, and $\mathrm{P}$ is the shock pressure. We accelerate our projectiles with a two stage gas gun, initially propelled by gunpowder. The variation in powder burning produces a final projectile velocity variation of $10 \%$ in some cases. Because of the high exponent of Eq. (1), this yields a variability in signal intensity of $\sim 2: 1$. Unknown sample equation of state may also contribute a significant uncertainty through the variation in $\mathrm{P}$, through $\mathrm{I} \sim \mathrm{T}^{4} \sim \mathrm{P}^{4}$. Because the useful portion of the signal lies at the top of a pulse, any overloading is a catastrophic loss of the catchup signature. This forces us to be especially conservative in choosing the nominal vertical gain of our digitizing recorder. However, the 8 bit vertical resolution is insufficient at the reduced nominal level to determine the catchup time to the desired precision.

As a solution, we have invented ${ }^{4}$ a simple method to eliminate the catastrophic sensitivity to clipping. The signal is recorded through a high pass circuit. The inverse operation is applied numerically during data analysis. We will demonstrate that the method is robust to clipping and preserves the high frequency information needed to determine the change of slope at the catchup point. As a generic method, it can be applied to any pulse experiment where the pertinent information lies on top of a roughly flat pulse.

\section{METHOD}

Our optical signal is detected with photomultipliers (PMT). Coaxial cables lead signals to Tektronix DSA 602 transient digitizers. Coaxial cable delays are used to increase the total cable length to $300 \mathrm{~ns}$ to make the appearance of spurious echos obvious. The vertical resolution of the digitizers is 8 bits, or 256 vertical points. The sampling interval is $2 \mathrm{~ns}$.

The traditional way to handle signals of high dynamic range is to use redundant channels with different vertical gain settings. We assign 2 PMTs to observe each of 6 target locations, with a high and low optical attention preceding each PMT tube. We must be mindful that in spite of the increased dynamic range of the digitizer using this method, the PMTs have their own dynamic range limitation.
Excessive light will cause PMT saturation. However, this saturation is more forgiving than the clipping of the digitizer. The change in slope at the catchup point can still be observed in the saturated signal, albeit weaker.

Our method ${ }^{4}$ is based on the idea that the catchup point is a change of slope, from a slope that is initially small. Therefore, if one records the derivative of the signal, the signal immediately preceding the catchup will always be near zero, and this can be placed at the center of the digitizing range for any gain.
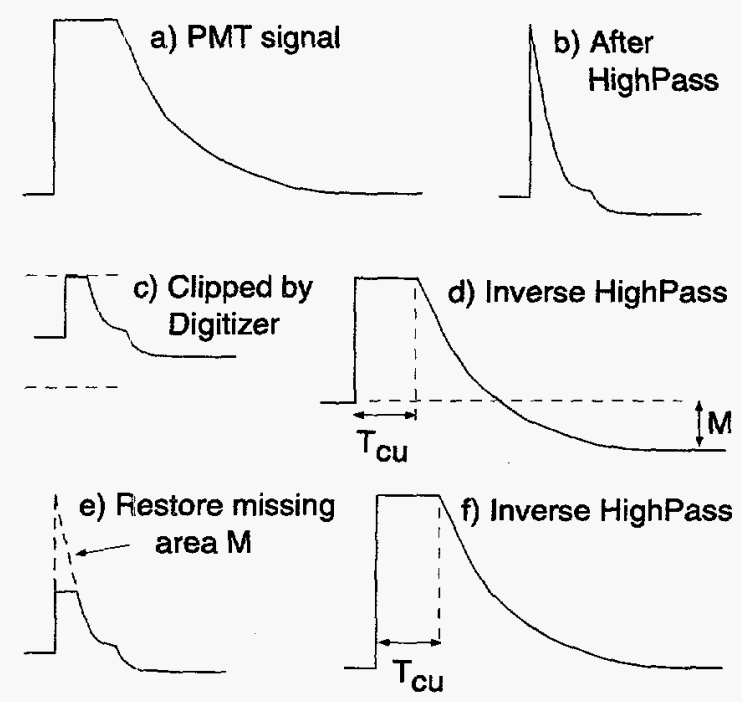

Figure 3. Steps in recording the experimental signal. a) Photomultiplier signal. b) After high pass circuit. c) Digitizer clips signal. d) After numerically applying inverse of high pass filter. Catchup time $T_{c u}$ can be obtained from this signal. Optionally, further processing will restore proper shape to tail. Baseline offset $M$ is the area that needs to be restored to derivative signal e). f) final result after applying inverse of high pass filter to e).

We can generate the derivative-like signal by a high pass circuit. This is trivially implemented by inserting a small capacitor in series at the digitizer input (Fig. 2). The $50 \Omega$ impedances of the digitizer input and cable with the capacitor form a high pass network with a time constant $\mathrm{T}_{\mathrm{rc}} \approx 30 \mathrm{~ns}$. Due to stray inductances, the behavior of the filter is more complex than a simple RC circuit. We do not attempt to model its effect by circuit theory. Instead, we measure the actual transfer function for the filter of each channel. This is done both 
accurately and conveniently by fast Fourier transforms of the digitized signals.

An electrical test pulse is applied to each channel, with and without the high pass filter and recorded by the digitizer as $V_{\text {with }}(t)$ and $V_{\text {without }}(t)$, respectively. The Fourier transforms $(f f t)$ are divided to produce the frequency response of the filter

$$
\operatorname{HighPass}(f)=\frac{f f t V_{\text {with }}(t)}{f f t V_{\text {without }}(t)} .
$$

If $V_{P M T}(t)$ is the PMT signal to be found, and $V_{\operatorname{rcrd}}(t)$ is the recorded digitizer signal, then without clipping the PMT signal is obtained

$$
f f t V_{P M T}(t)=\frac{f f t V_{r c r d}(t)}{H i g h P a s s(f)} \text {. }
$$

The steps in the recording process are illustrated in Fig. 3. The source PMT signal a) is converted to a derivative-like signal $b$ ) by the high pass circuit. This signal suffers clipping, because a high gain is deliberately used to improve the signal to noise ratio around the catchup point. We have determined that large amounts of clipping can be tolerated by the process without detrimenting the catchup signature, as long as the catchup point is not closer than about $\mathrm{T}_{\mathrm{rc}}$ to the pulse start.

After the experiment, the recorded derivativelike signal is "integrated" by dividing its Fourier transform by HighPass(f) by Eq. (3). This signal (Fig. 3d) is sufficient to determine the catchup point, since all the high frequency information, except under the clipped region, is present. However, for mostly cosmetic reasons, the signal can be further processed to bring a closer resemblance to the original in low frequencies as well. The difference in baselines (Fig. 3d) is defined as $\mathrm{M}$. A triangle of area $\mathrm{M}$ is added to the clipped signal (Fig. 3e). When this is integrated via Eq. (3), the result Fig. $3 f$ is very close to the original in both low and high frequency information.

The only portion of the signal significantly altered by the clipping is directly under the clipped region, which is approximately a time constant $\mathrm{T}_{\text {rc }}$ from the pulse start. We have numerically tested the ability to resolve the catchup point close to the pulse start on the severity of clipping. For the PMT pulse, we used an experimental signal (Fig. $4 \mathrm{a}$, bold) measured previously with direct coupling between the PMT and digitizer. The simulated derivative-like signal (Fig. 4b, bold) was obtained by applying Eq. (3), and artificially clipped at either of two levels. This was then integrated via Eq. (3) and shown as light curves against the original curve in Fig. 4a).
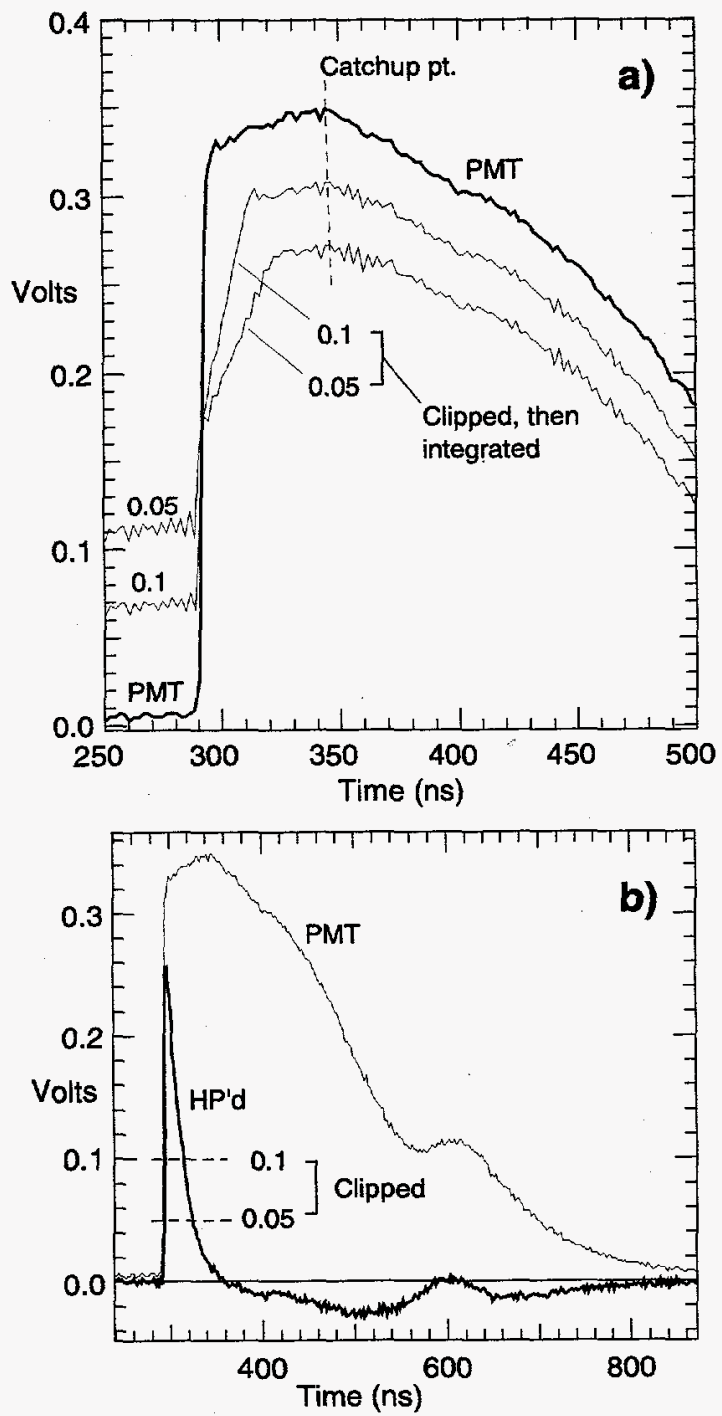

Figure 4. Effect of clipping on reconstituted signal is tested numerically. a) Bold curve: source PMT signal. b) Bold: simulated highpassed signal obtained by multiplying Fourier transform of PMT by HighPass(f), which was measured from an actual filter. Signal was then clipped at either level 0.1 or 0.05 and integrated by division of its Fourier transform by HighPass $(f)$. These reconstituted curves are light curves in a). Curves shifted vertically for clarity. Data for a short time after shock is altered, but change in slope at catchup point remains.

The results show that this method is very robust to severe clipping. Only the region directly clipped 
was affected. All other regions retained the high frequency information content needed for detecting the change in slope.

We observe that our digitizer does not appear to be "blinded" by overloading; it recovers promptly.

\section{CONCLUSION}

This method improves the signal to noise ratio of the catchup portion of the signal. By preventing catastrophic loss of data through clipping, the nominal signal level can be increased to a greater level than would otherwise be advisable.

\section{ACKNOWLEDGMENT}

This research was performed under the auspices of the U.S. Department of Energy by the Lawrence Livermore National Laboratory under contract W7405-Eng-48.

\section{REFERENCES}

1. R. G. McQueen, J. W. Hopson, and J. N. Fritz, Rev. Sci. Instsrum. 53, 245 (1982)

2. R. G. McQueen, D. G. Isaak, in "Shock Compression of Condensed Matter-1989", Ed. S. C. Schmidt et al., (Elsevier Science Pub., NY 1990), p. 125.

3. J. N. Fritz, C. E. Morris, R. S. Hixson, and R. G. McQueen in "High-Pressure Science and Technology-1993", Ed. S.

C. Schmidt et al., (AIP Press, NY 1994), p. 149.

4. D. J. Erskine, Rev. Sci. Instr. Sept. 1995. 